\title{
REVIEW \\ Animal models of spinal cord injury: a systematic review
}

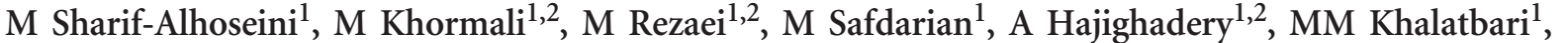 \\ M Safdarian ${ }^{1}$, S Meknatkhah ${ }^{3}$, Mezvan $^{1}$, M Chalangari ${ }^{1}$, P Derakhshan ${ }^{1}$ and V Rahimi-Movaghar ${ }^{1}$
}

Study Design: PRISMA-guided systematic review.

Objectives: To provide a comprehensive framework of the current animal models for investigating spinal cord injury (SCl) and categorize them based on the aims, patterns and levels of injury, and outcome measurements as well as animal species.

Setting: Sina Trauma and Surgery Research Center, Tehran University of Medical Sciences, Tehran, Iran.

Methods: An electronic search of the Medline database for literature describing animal models of SCI was performed on 1 January 2016 using the following keywords: 'spinal cord injuries' and 'animal models'. The search retrieved 2870 articles. Reviews and non-original articles were excluded. Data extraction was independently performed by two reviewers.

Results: Among the 2209 included studies, testing the effects of drug's or growth factor's interventions was the most common aim (36.6\%) followed by surveying pathophysiologic changes (30.2\%). The most common spinal region involved was thoracic $(81 \%)$. Contusion was the most common pattern of injury (41\%) followed by transection (32.5\%) and compression (19.4\%). The most common species involved in animal models of $\mathrm{SCl}$ was the rat (72.4\%). Two or more types of outcome assessments were used in the majority of the studies, and the most common assessment method was biological plus behavioral (50.8\%).

Conclusions: Prior to choosing an animal model, the objectives of the proposed study must precisely be defined. Contusion and compression models better simulate the biomechanics and neuropathology of human injury, whereas transection models are valuable to study anatomic regeneration. Rodents are the most common and probably best-suited species for preliminary SCl studies.

Spinal Cord (2017) 55, 714-721; doi:10.1038/sc.2016.187; published online 24 January 2017

\section{INTRODUCTION}

Spinal cord injury (SCI), a fundamental medical problem, is associated with severe disability and high rates of mortality. Although there is no definite treatment for SCI, various research studies are ongoing including experimental models to understand the anatomical and biological events involved in $\mathrm{SCI}$ and repair, and to test the safety and efficacy of potential therapies. Animal models are in vivo investigational methods used to analyze events under controlled conditions. Therefore, to be insightful for a given human condition, a disease or injury model should not only be similar in terms of the causation and function to the human analog but also must have advantages over simple clinical observation. ${ }^{1,2}$ Animal models also allow for in-depth investigation of physiological and pathological events. An astute researcher should be capable of choosing a proper model and outcome assessment given the specific objectives. To select an appropriate model and test a specific hypothesis, all existing SCI animal models and related outcome assessments must be considered. So far, some reviews focused on the paradigms ${ }^{3,4}$ or outcomes ${ }^{5-7}$ in animal models of SCI. However, there is a gap in the literature for a complete categorization of animal models of SCI studies. This systematic review provides a comprehensive framework of the current animal models of SCI and categorizes them based on the study aims, patterns and levels of injury, and outcome measurements as well as animal species.

\section{MATERIALS AND METHODS}

This systematic review was conducted according to the Preferred Reporting Items for Systematic Reviews and Meta-analysis (PRISMA) statement. ${ }^{8}$ An electronic search of the Medline literature for animal models of SCI was performed (1946 to 1 January 2016), using the following conditions: SCI (MeSH Terms) AND (Models, Animal (MeSH Terms) OR Behavior, Animal/Physiology (MeSH Terms) OR Animal Experimentation (MeSH Terms)). Next the titles and abstracts of studies of the resultant articles were independently screened by two reviewers. Reviews and non-original articles were excluded at this stage. Full-texts of the remaining manuscripts were retrieved and further screened by reviewers against the exclusion/inclusion criteria without language restriction. Reports were excluded based on the following: review articles, articles not relevant to the experimental SCI, in vitro spinal cord preparations and overlapping publications of larger studies. In the latter case, the original studies that had used animal models of SCI were included in the systematic review. Data extraction from the resulting studies meeting inclusion criteria was performed. The purpose of data extraction was to obtain the goals, methods and outcome measurements from each study in a consistent manner so that the findings could be interpreted and analyzed (Supplementary 1). The data extraction form was pre-tested by all investigators using five sample studies. Agreement among reviewers in this pilot test was achieved by discussion to expose any ambiguity of the instrument and

${ }^{1}$ Sina Trauma and Surgery Research Center, Tehran University of Medical Sciences, Tehran, Iran; ${ }^{2}$ Students' Scientific Research Center, Tehran University of Medical Sciences, Tehran, Iran and ${ }^{3}$ Laboratory of Neuro-Organic Chemistry, Institute of Biochemistry and Biophysics (IBB), University of Tehran, Tehran, Iran

Correspondence: Professor V Rahimi-Movaghar, Sina Trauma and Surgery Research Center, Tehran University of Medical Sciences, Sina Hospital, Hassan-Abad Square, Imam Khomeini Avenue, Tehran, 11365-3876, Iran.

Email: v_rahimi@sina.tums.ac.ir

Received 10 July 2016; revised 8 November 2016; accepted 27 November 2016; published online 24 January 2017 
to improve subsequent reliability. Extraction was executed by the reviewers in the following manner: a reviewer would first extract data into the form and a second reviewer would confirm the accuracy of data extracted by the first reviewer. Any disagreements were resolved by a third reviewer. Full-text of studies in languages other than English would be screened by one reviewer only. For this purpose, the corresponding authors of non-English articles were asked to send the English version of research method via email. In case that no reply was received, translations were done using Google Translate. Descriptive summaries were conducted using SPSS (IBM Corp., Endicott, NY, USA).

\section{RESULTS}

Initial records identified through Medline literature searching included 2870 articles (Supplementary 2). The study selection procedure is summarized in Figure 1.

In total, 661 articles were excluded because they were reviews, non-original articles, not related to the experimental SCI, or a part of larger included studies. A total of 74 non-English articles were included.

Among the 2209 included studies, testing the effects of drug's or growth factor's interventions was the most common aim of studies (36.6\%) followed by evaluating pathophysiologic changes (30.2\%). Other aims and their frequencies based on injury patterns are shown in Table 1 .

The most common spinal region studied was thoracic $(1790,81 \%)$ followed by cervical $(265,12 \%)$, lumbar $(113,5.1 \%)$, sacral $(16,0.7 \%)$, other $(16,0.7 \%)$ and unknown $(64,2.9 \%)$. In 54 studies $(2.4 \%)$, SCIs were induced in multiple regions.

On the basis of the mechanism of injury, SCI models were classified as mechanical traumatic injury (94.5\%) and non-mechanical injury (5.5\%). Contusion as a transient force to displace and damage the spinal cord was the most common pattern of injury. Transection and compression which were characterized by compression of the spinal cord over an extended period of time were the next most frequent injury patterns. In 25 studies (1.1\%), more than one pattern was used. Figure 2 shows all the types and subtypes of mechanical traumatic SCI patterns in 2087 related studies.

The injury extent was not reported in 722 studies (33\%). In 785 studies (35\%), the injury was described as complete, and incomplete injury was used in another 702 studies (32\%), which included transection.

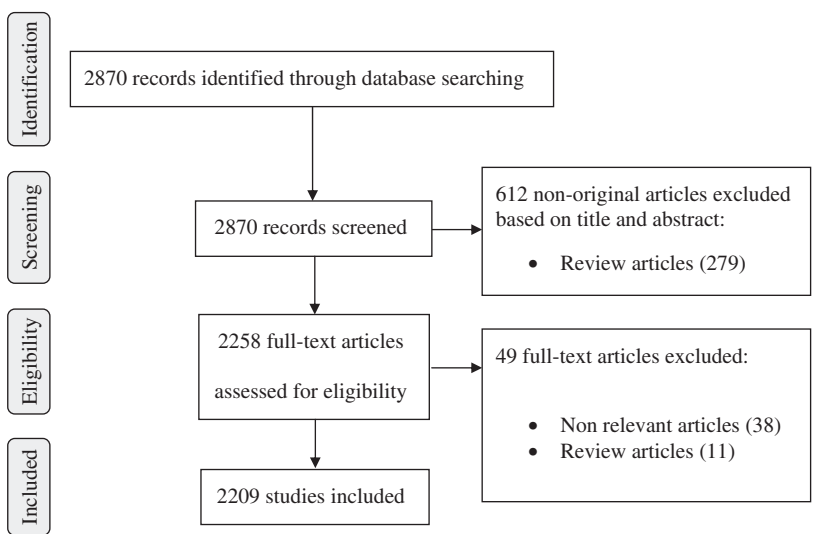

Figure 1 Flow diagram of summarized search procedure. A full color version of this figure is available at the Spinal Cord journal online.

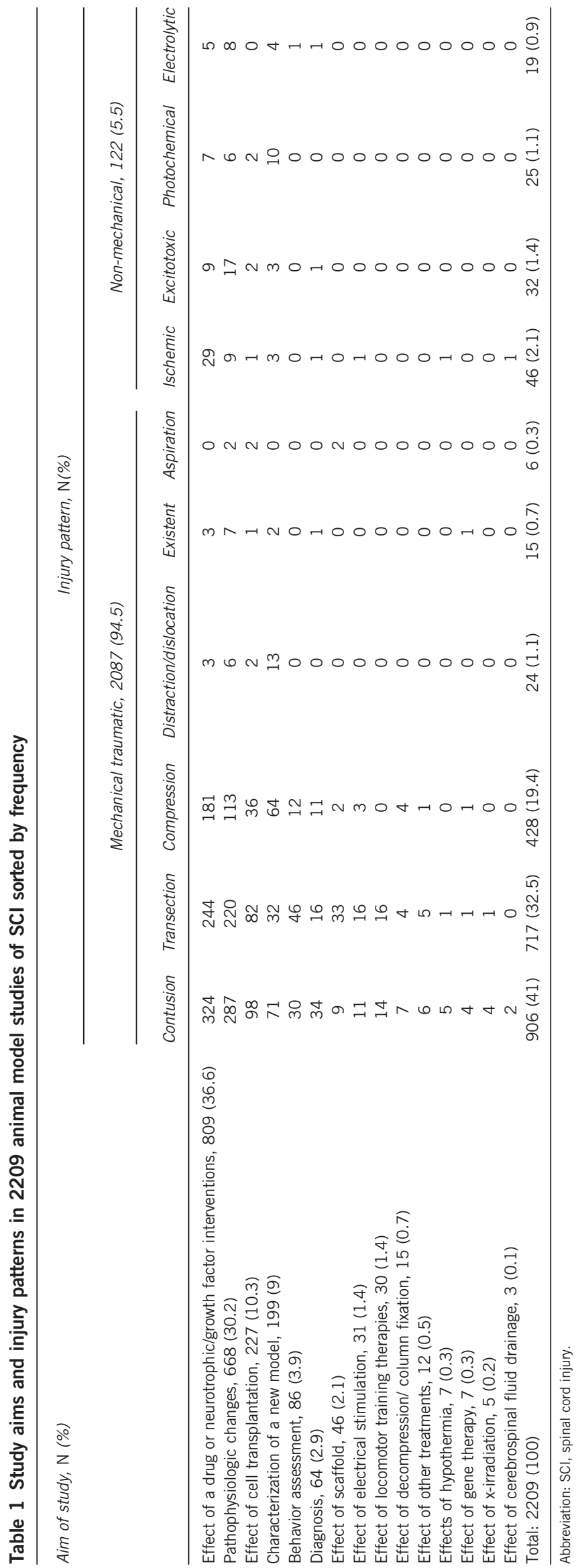




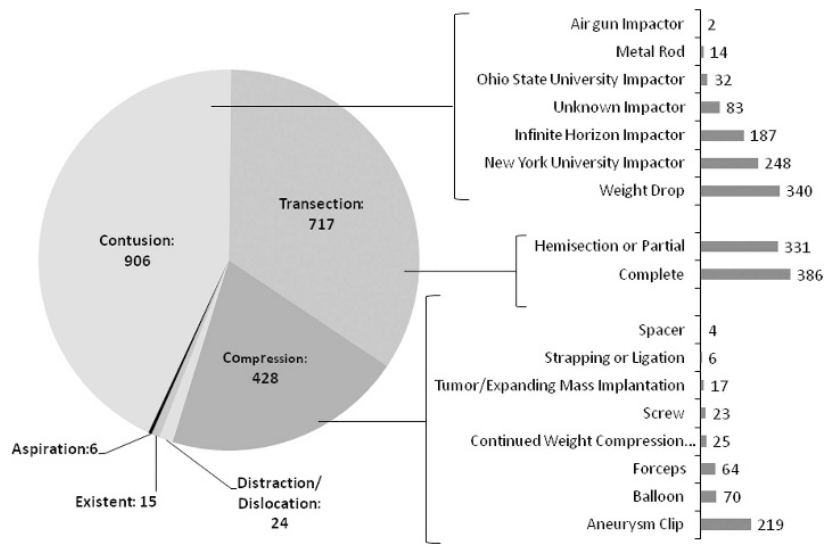

Figure 2 Injury types and subtypes in 2087 animal model studies with mechanical traumatic $\mathrm{SCl}$. A full color version of this figure is available at the Spinal Cord journal online.

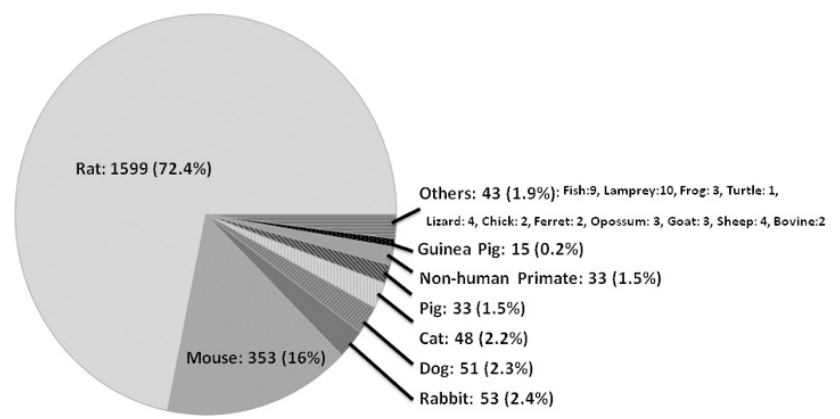

Figure 3 Species of animal used in 2209 animal model studies of SCl.

Rat was the most common species in the animal models of SCI. All species of animal used in studies are shown in Figure 3.

Outcome assessments were classified into seven categories which included biological, behavioral, neurophysiology, imaging, musculoskeletal, cardiovascular and respiratory evaluations (Table 2, Supplementary 3). Figure 4 is a schematic drawing of outcome assessments based on frequency and coincidence. With respect to the histological methods, chemistry techniques and behavioral methods used, these were expanded based on the specific tests, frequencies and features presented in Tables 3 and 4, separately.

Sixty-three percent of studies used two or more types of outcome assessments (Table 5). The most common combination of multiple outcome assessments was the usage of biology and behavior assessments.

\section{DISCUSSION}

\section{Aim of studies}

Testing the effects of drug's or growth factor's interventions was the most common aim of studies (36.6\%) followed by evaluating pathophysiologic changes (30.2\%). Because of the technical and ethical issues associated with human studies, most of the existing knowledge regarding SCI pathophysiology and related interventions is derived from animal studies. ${ }^{9}$ Animal models provide opportunities to discern the cause and effect relationship between functional deficits and their essential biology. However, the heterogeneous nature of SCIs remains an obstacle to translating findings across different paradigms and severity of injuries as well as various species and to humans. ${ }^{10}$ Table 1 , showing the aims of studies based on injury patterns, could help
Table 2 Outcome assessments in $\mathbf{2 2 0 9}$ animal model studies of SCI sorted by frequency ${ }^{a}$

\begin{tabular}{|c|c|}
\hline Type, N (\%) & Subtype, $\mathrm{N}(\% \text { of type })^{\mathrm{a}}$ \\
\hline Biology, 1827 (82.7) & $\begin{array}{l}\text { Immunohistochemistry, } 1154(63.2) \\
\text { Staining and labeling }{ }^{\mathrm{b}}, 1089 \text { (59.6) } \\
\text { Chemistry techniques }^{\mathrm{b}}, 644(35.2) \\
\text { Genetic techniques, } 371 \text { (20.3) } \\
\text { Flow cytometry, } 42(2.3) \\
\text { Blood-spinal cord barrier permeability, } 34 \text { (1.9) } \\
\text { Cell Count, } 30 \text { (1.6) }\end{array}$ \\
\hline Behavior $^{c}, 1383$ (62.6) & $\begin{array}{l}\text { Locomotor tests, } 1234 \text { (89.2) } \\
\text { Sensory tests, } 225 \text { (16.3) } \\
\text { Sensory-motor tests, } 184 \text { (13.3) } \\
\text { Autonomic tests, } 92 \text { (6.7) } \\
\text { Reflex-response based tests, } 78(5.6)\end{array}$ \\
\hline Neurophysiology, 393 (17.8) & $\begin{array}{l}\text { SSEPs, } 162 \text { (41.3) } \\
\text { Motor-evoked potentials, } 129 \text { (32.8) } \\
\text { Electromyography, } 113(28.8) \\
\text { Electroneurogram, } 39(9.9) \\
\text { Membrane potentials, } 29(7.4)\end{array}$ \\
\hline Imaging, 151 (6.8) & $\begin{array}{l}\text { MRI, } 108 \text { (71.5) } \\
\text { Computerized tomography, } 19 \text { (12.6) } \\
\text { Functional MRI, } 11 \text { (7.3) } \\
\text { X-ray, } 8 \text { (5.3) } \\
\text { Ultrasonography, } 6 \text { (4) } \\
\text { Positron emission tomography, } 4 \text { (2.6) }\end{array}$ \\
\hline
\end{tabular}

Cardiovascular evaluation, 61 (2.8)

Musculoskeletal evaluation, 41 (1.9)

Respiratory evaluation, 17 (0.8)

Abbreviations: MRI, magnetic resonance imaging; SCI, spinal cord injury; SSEP, somatosensory evoked potentials.

aSome studies used more than one outcome assessment, simultaneously (See Table 5). bexpanded in Table 3.

'Expanded in Table 4.

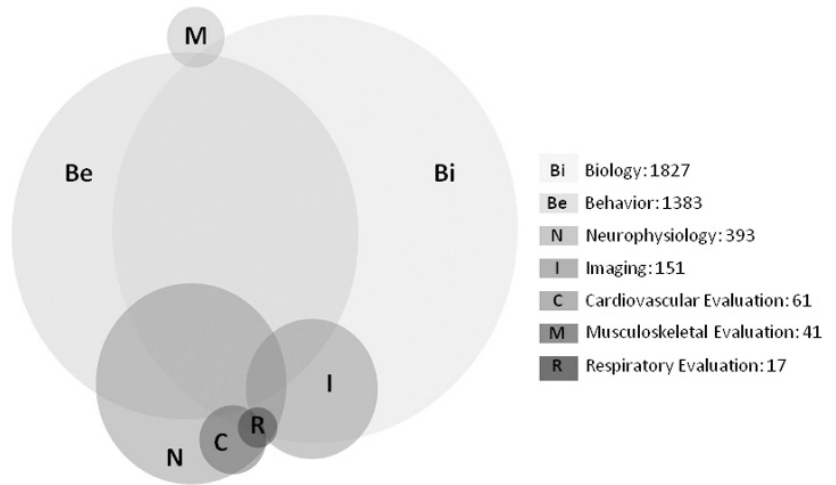

Figure 4 Schematic drawing of outcome assessments based on frequency and coincidence in 2209 animal model studies of SCl.

researchers to select an appropriate model, especially about less common and non-mechanical models.

\section{Region of injury}

The most common region involved in SCIs in humans is the cervical. ${ }^{11}$ However, these data indicate that thoracic SCIs are the most common location used in animal models (81\%). On the basis of the literature, thoracic SCI models are apparently reliable and easy to reproduce. ${ }^{12,13}$ As reduction of gray matter in this spinal region causes less identifiable functional loss, SCIs in thoracic levels permit the 
Table 3 Staining and Labeling, and Chemistry Techniques in 2209 animal model studies of SCl, classified using MeSH database and sorted by frequency

\begin{tabular}{|c|c|c|}
\hline Main technique, $\mathrm{N}(\%)$ & Specific technique, N (\% of main technique) & Reflects \\
\hline \multirow[t]{12}{*}{ Staining and labeling, 1089 (49.3) } & H\&E, 511 (46.9) & Spared tissue \\
\hline & Luxol fast blue, 229 (21) & Myelin \\
\hline & Cresyl violet, 223 (20.5) & Nissl bodies \\
\hline & Tract tracing, 216 (19.9) & Neural pathways \\
\hline & Osmium tetroxide, 210 (19.3) & Myelin, for electron microscopy \\
\hline & Eriochrome (solochrome) cyanine, 75 (6.9) & Myelin \\
\hline & Toluidine blue, 46 (4.2) & Nissl bodies \\
\hline & Silver, 35 (3.2) & Axon distribution and vascularity \\
\hline & Masson's Trichrome, Van Gieson or Sirius Red, 17 (1.6) & Collagen fibers \\
\hline & Kluver-Barrera or Weil, 16 (1.5) & Myelin \\
\hline & In Situ hybridization, 15 (1.4) & Nucleic acid \\
\hline & Prussian blue, 4 (0.4) & Iron in the tissue \\
\hline \multirow[t]{5}{*}{ Chemistry techniques, 644(29.2) } & Identification of proteins (western blotting, electrophoresis), 480 (74.5) & Protein interactions \\
\hline & Peroxidase activity, 110 (17.1) & Leukocyte accumulation \\
\hline & Biochemical analysis, 103 (16) & Chemical reactions or enzymatic activities \\
\hline & High performance liquid chromatography, 23 (3.6) & Substance P \\
\hline & Cerebrospinal fluid analysis, 18 (2.8) & Cerebrospinal fluid properties \\
\hline
\end{tabular}

Abbreviations: $\mathrm{H} \& \mathrm{E}$, hematoxylin and eosin; SCl, spinal cord injury.

isolation and study of white matter deficits. ${ }^{14}$ On the other hand, cervical SCIs can result in respiratory compromise and are associated with a high mortality rate due to interruption of the bulbospinal respiratory drive to phrenic motoneuron pools and diaphragm impairment. ${ }^{14,15}$ However, cervical and thoracic spinal levels differ with respect to various anatomical aspects such as spinal cord diameter, distance between the cell bodies of injured axons and the injury site, relative dedication of the cord to specific ascending and descending pathways, degree of vascularization, size of the sensory and motor neuron populations and white/gray matter composition. ${ }^{16}$ Therefore, thoracic SCI models may not be readily translatable to cervical injury patterns. ${ }^{16}$ In the cervical region, white matter disruption leads to spastic paralysis below the level of injury, sensory loss and chronic pain, as well as gastrointestinal, cardiovascular and sexual dysfunction. ${ }^{11}$ Furthermore, use of the hind limb paws and digits cannot be assessed as carefully as in the forelimb. Thus, forelimb evaluation could overestimate the efficacy of potential therapies, particularly when improvements are mild. ${ }^{16,17}$ These differences and region-specific properties justify the development of cervical SCI models in recent years. ${ }^{18}$ On the basis of these data, however, cervical SCI was done in only $12 \%$ of the studies considered.

\section{Pattern of injury}

In general, SCI models are selected based on the aims of the researchers. Every animal model and injury pattern is designed to focus on a specific question, and hence has associated consideration, advantages and disadvantages. Most human SCIs happen due to blunt trauma (for example, motor vehicle crash), where the spinal cord is damaged by an object or displaced bone or other tissue. On the basis of our data, $62 \%$ of included studies used a blunt trauma injury pattern such as contusion, compression, distraction, dislocation or existing traumatic SCI (for example, dogs injured in traffic accidents; Table 2). These models were developed in order to study post-traumatic lesional processes, and repair processes after spinal cord decompression or neuroprotective treatment. ${ }^{19}$

If the aim of study is investigation of pathophysiologic changes, a contusion and/or compression model is preferred, because most human SCIs involve contusion or compression..$^{20}$ Contusion is the oldest and most commonly used for SCI models. The first reported SCI model was presented by Allen in 1911, where a mass was dropped from a prescribed height onto the dorsal surface of the canine dura. Most data available concerning induction of a contusive SCI are modifications of the injury model proposed by Allen. ${ }^{21}$ The contusive models can make SCI in several severities which have been characterized by inflammation, ischemia, hemorrhagic necrosis and central cavitation. Our study showed that Weight Drop was the most common method of contusion (37.5\%), followed by New York University (NYU) impactor (27.4\%) and infinite horizon impactor (20.6\%). Although the New York University impactor is rather easier to use, due to electromagnetic driving the infinite horizon and Ohio State University impactors have greater precision and more reliably produce SCIs. ${ }^{22}$ Unilateral contusion or hemicontusion is typically used in cervical regions because life-threatening adverse effects could occur after complete cervical lesions. As motor dysfunction is more apparent in the forelimbs, pain-related behavior is difficult to estimate, and hence cervical contusion is frequently utilized only for motor functional analysis. ${ }^{11}$

Compression models are helpful to simulate the constant spinal canal occlusion that is common in human SCIs and study the effects of compression or the optimal timing of decompression. Appropriate devices for compressive SCI are shown in Table 2. On the basis of our data, more than half of the compression models use the aneurism clip that causes ischemia and mimics common clinical injuries. ${ }^{23,24}$ The balloon-induction method is a simple technique that does not cause any damage to the surrounding structures. ${ }^{25}$ Calibrated forceps can be used to produce a lateral compression injury by insertion on either side of the spinal cord. ${ }^{1}$ A spacer can produce a precise degree of narrowing of the spinal canal and mimic an anteroposterior compression. ${ }^{26}$

Dislocation models cause injury by lateral displacement of vertebra, whereas distraction models apply opposing traction forces to stretch the spinal cord. ${ }^{4}$

Transection models are useful to study the effects of scaffolds, biomaterials and neurotrophic factors in SCI and may be more appropriate to investigate regeneration, degeneration, tissue engineering strategies or neuroplasticity. ${ }^{27}$ Sometimes transection models are 
Table 4 Behavior assessment tests in 2209 animal model studies of SCl sorted by frequency

\begin{tabular}{|c|c|c|}
\hline Main test, $\mathrm{N}(\%)$ & Specific test, $\mathrm{N}$ (\% of main test) & Reflects \\
\hline \multirow[t]{20}{*}{ Locomotor, 1234 (55.9) } & BBB, 609 (49.3) & Locomotion \\
\hline & Open-field activity, 200 (16.2) & Locomotion \\
\hline & Inclined plane, 145 (11.8) & Muscle strength \\
\hline & Basso mouse scale, 115 (9.3) & Locomotion \\
\hline & Automated walkway, 86 (7) & Locomotion \\
\hline & Tarlov, 81 (6.6) & Locomotion \\
\hline & Kinematic analysis during treadmill, 74 (6) & Locomotion \\
\hline & Forelimb locomotor scale, 66 (5.3) & Locomotion \\
\hline & Footprint, 55 (4.5) & Motor coordination \\
\hline & Hind limb function, 41 (3.3) & Locomotion \\
\hline & Swim, 36 (2.9) & Swimming ability \\
\hline & Limb grip strength, 36 (2.9) & Muscle strength \\
\hline & Food pellet reaching, 36 (2.9) & Motor coordination \\
\hline & Forelimb asymmetry, 31 (2.5) & Paw preference \\
\hline & Rotarod, 31 (2.5) & Locomotion \\
\hline & Combined behavioral score, 29 (2.4) & Locomotion \\
\hline & Rearing, 25 (2) & Paw preference \\
\hline & Limb hanging, 14 (1.1) & Grasping \\
\hline & Tail position, $4(0.3)$ & Locomotion \\
\hline & Eshkol-Wachmann notation, 1 (0.1) & Locomotion \\
\hline \multirow[t]{5}{*}{ Sensory, 225 (10.2) } & Von Frey or Semmes-Weinstein, 128 (56.9) & Mechanical allodynia \\
\hline & Pinch reflex, 109 (48.4) & Reflex \\
\hline & Hot plate, 58 (25.8) & Temperature \\
\hline & Cold tests, 17 (7.6) & Temperature \\
\hline & Paw compression, 12 (5.3) & Pain \\
\hline \multirow[t]{5}{*}{ Sensory-motor, 184 (8.3) } & Grid walking (foot fault), 80 (43.5) & Sensory-motor coordination \\
\hline & Foot slip (horizontal ladder), 61 (33.2) & Sensory-motor coordination \\
\hline & Grooming, 28 (15.2) & Sensory-motor connection \\
\hline & Narrow beam walking, 28 (15.2) & Balance \\
\hline & Rope walk testing, 10 (5.4) & Balance \\
\hline \multirow[t]{6}{*}{ Autonomic, 92 (4.2) } & Urinary bladder function, 69 (75) & Bladder function \\
\hline & Autonomic dysreflexia, 13 (14.1) & Autonomic dysreflexia \\
\hline & Erection-based tests or mating, 6 (6.5) & Erection \\
\hline & Telemetric monitoring, 6 (6.5) & Micturition erection \\
\hline & Manometric or balloon Test, 4 (4.3) & Sphincter control \\
\hline & Gastric motility, 3 (3.3) & Gastric function \\
\hline \multirow[t]{4}{*}{ Reflex-response, 78 (3.5) } & Contact placing response, 43 (55.1) & Reflex \\
\hline & Toe spread reflex, 28 (35.9) & Reflex \\
\hline & Righting reflex (Static), 18 (23.1) & Reflex \\
\hline & Hoffmann's reflex, 12 (15.4) & Reflex \\
\hline
\end{tabular}

Abbreviations: BBB, Basso, Beattie, Bresnahan scale; SCI, spinal cord injury.

Table 5 Applied types of outcome assessments in 2209 animal model studies of SCl

\begin{tabular}{ll}
\hline $\begin{array}{l}\text { Types of outcome } \\
\text { assessments, N (\%) }\end{array}$ & The most common mode, N (\%) \\
\hline One type, $823(37.3)$ & Biology, $574(26)$ \\
Two types, $1122(50.8)$ & Biology+behavior, $886(40.1)$ \\
Three types, $245(11.1)$ & Biology+behavior+neurophysiology, 148 (6.7) \\
Four types, $19(0.9)$ & Biology+behavior+neurophysiology+imaging, $18(0.8)$ \\
\hline
\end{tabular}

Abbreviation: $\mathrm{SCl}$, spinal cord injury.

used to study the role of neuronal circuitries in locomotion. ${ }^{28-31}$ On the other hand, transected spinal cords are rarely encountered in clinical practice, and thus the transection models do not truly reflect the pathophysiology of SCI and are not appropriate as a basis to test neuroprotective treatment. ${ }^{32}$ Spinal cord transection is performed after laminectomy with fine surgical scissors (iridectomy scissors) to induce complete or targeted interruption of the spinal cord. ${ }^{22}$ Therefore to maintain tissue continuity, a researcher can interrupt selective pathways via partial transections and preserve a tissue connection between the rostral and caudal ends of the spinal cord. ${ }^{33}$ On the basis of our study, almost half of the transection models are incomplete or partial. (Table 2) Since a complete transection makes such a destructive tissue milieu, incomplete transection reduces the physical harm to the cord and the major cavitation and physical separation. Several studies have revealed different plasticity in the spinal cord after complete and incomplete transections. ${ }^{31}$ Therefore, the researchers should be careful in the interpretation results focusing the extent of injury. A dorsal hemisection for selective interruption of the corticospinal tract can be performed with some feedback from the change in texture and color between the white and gray matter, giving an indication of the degree of hemisection. ${ }^{1,34}$ However, dorsal hemisection cannot be used specifically to evaluate true axon regeneration. ${ }^{33}$ Dorsolateral quadrant 
Table 6 The three most commonly used outcome assessments in rat model studies with cervical and thoracic $\mathrm{SCI}$

\begin{tabular}{|c|c|c|c|c|c|c|c|}
\hline \multirow[t]{3}{*}{ Level of Injury } & \multirow[t]{3}{*}{ Injury Pattern } & \multicolumn{6}{|c|}{ Outcome assessments } \\
\hline & & \multicolumn{3}{|c|}{ Biology } & \multicolumn{3}{|c|}{ Behavior } \\
\hline & & 1 & 2 & 3 & 1 & 2 & 3 \\
\hline \multirow[t]{3}{*}{ Cervical } & Transection & $\mathrm{IHC}$ & Tract tracing & Cresyl violet & $\begin{array}{l}\text { Food pellet } \\
\text { reaching }\end{array}$ & $\begin{array}{l}\text { Forelimb } \\
\text { asymmetry }\end{array}$ & $\begin{array}{l}\text { Kinematic analysis during treadmill } \\
\text { locomotion }\end{array}$ \\
\hline & Contusion & $\mathrm{IHC}$ & Cresyl violet & $H \& E$ & BBB & Limb grip strength & Forelimb asymmetry \\
\hline & Compression & $\mathrm{IHC}$ & $H \& E$ & Tract tracing & BBB & $\begin{array}{l}\text { Food pellet } \\
\text { reaching }\end{array}$ & $\begin{array}{l}\text { Forelimb asymmetry or Limb grip } \\
\text { strength }\end{array}$ \\
\hline \multirow[t]{3}{*}{ Thoracic } & Transection & $\mathrm{IHC}$ & $\begin{array}{l}\text { Identification of } \\
\text { proteins }^{a}\end{array}$ & Tract tracing & BBB & Open-field activity & Inclined plane \\
\hline & Contusion & $\mathrm{IHC}$ & Identification of proteins & $H \& E$ & BBB & Open-field activity & Inclined plane \\
\hline & Compression & $\mathrm{IHC}$ & $H \& E$ & Identification of proteins & BBB & Open-field activity & Inclined plane \\
\hline
\end{tabular}

Abbreviations: BBB, Basso, Beattie, Bresnahan scale; H\&E, hematoxylin and eosin; IHC, immunohistochemistry; SCI, spinal cord injury.

Western blotting, electrophoresis.

lesions are used to disrupt the rubrospinal tract, and lateral hemisections interrupt all tracts on one side while sparing some or all tracts on the opposite side. The transection model is the most appropriately combined with neuroanatomical tract tracing and electrophysiological studies. $^{22,34}$

Ischemic models are established using an anterior sternotomy with temporary aortic occlusion created by aneurysm clips. Spinal cord ischemia remains an unknown clinical dilemma which mostly occurs after aortic problems. ${ }^{35-37}$ The present study showed that more ischemic models $(63 \%)$ were performed to the test effects of drug's or growth factor's interventions after SCI.

Excitotoxic models replicate selected components of the secondary injury cascade. They are produced through intraspinal or intrathecal injection of some excitotoxins. ${ }^{38}$

Photochemical models are among the most reproducible ischemic models of SCI. ${ }^{39}$ There is no need for laminectomy or mechanical trauma to the cord. After spinal column exposure and appropriate beam placement, a photosensitizing dye (for example, rose Bengal) is administrated systemically. The resultant photochemical reaction leads to vascular stasis and congestion, edematous pale-staining white matter tracts and hemorrhagic necrosis of the central gray matter. ${ }^{7}$

Electrolytic injury is used to study the neuronal pathways in the central nervous system. The technique involves the insertion of a fine metal electrode into the spinal cord. Restricted damage of cells in this region is accomplished by heating the electrode tip by passage of a high electric current. ${ }^{40}$ On the basis of our review, $84 \%$ of electrolytic injury models were composed in the spinothalamic tract of rats to study behavioral outcomes, especially with central neuropathic pain.

\section{Animal species}

Our analysis demonstrated that rodents were the most commonly used species (92\%; Figure 3). Although rodents are best suited for preliminary SCI studies, ${ }^{41}$ large animal and non-human primate experiments better approximate human SCIs. ${ }^{42}$ Nonetheless, due to expensive care and housing, rigorous regulatory requirements, and ethical considerations, larger mammals are rarely used. ${ }^{27}$ Other vertebrates, such as fish and lamprey, have been studied to test novel strategies for human SCI due to their distinctive regenerative capacities. $^{43}$

Rat models were the most widely used to study SCIs $(72.4 \%)$. The rat is low cost and easy to care for with a well-understood anatomy, relatively uncommon surgical infections, and wellestablished functional analysis techniques. ${ }^{7,34}$ Furthermore, similar to human pathology, rats often develop large cystic cavities at the site of injury. For these reasons, more rats are typically preferable to mimic the human pathology. ${ }^{44}$ However, rats are quadrupeds not bipeds, and their corticospinal tract is mainly dorsal, ${ }^{45}$ and these differences must be properly accounted for.

Mice models are being implemented increasingly to study the basic cellular and molecular biology of SCIs. ${ }^{44}$ These data estimate that $16 \%$ of SCI models used mice. Advantages of mice models include the similar genomes to humans, ease of handling, high reproductive rates and low cost of use. ${ }^{46}$ Conversely, the small working size prohibits many surgical maneuvres and device implantations. ${ }^{7,34}$ The injury site in mice is densely packed with cells and actually decreases in size over time due to absence of the cyst. Knockout models as genetically modified animal studies mainly use mice. Using rats for knockout models is so difficult. ${ }^{47}$

\section{Outcome assessments}

Seven types of outcome assessments and the rate of use of each type of the included studies are presented in Tables 2,3,4 and 5. Nearly two-thirds of these studies used two or more types of outcome assessments with the most common assessment being biology plus behavior tests (1122 studies, 50.8\%). Greater variety of outcome assessments improves the study significance and provides the possibility of observing changes from different perspectives. With respect to the testing methods used in 1528, rat models of mechanical traumatic SCI (as the most widespread models), the three most common outcome assessments, are presented in Table 6.

Biological analysis can yield helpful information on the extent of the primary injury, preservation of particular fiber tracts, cellular infiltration, secondary degeneration and molecular changes. At later stages it can be used to evaluate the scar and cyst formation. ${ }^{22}$ Besides traditional histological stainings, there is a broad variety of immunohistochemistry methods that allow for the investigation of cells, structures and processes. The cell phenotype expressing a particular protein in the injured spinal cords could be visualized using immunohistochemical analysis. Also, the identification of proteins is done to evaluate differential gene expression involved in SCI and treatment models thereof. Neuroanatomical tract tracing gives a detailed visualization of specific neuronal connections. Tract tracing 
demonstrates regeneration and plasticity after defined fiber lesions, and augments functional and physiological studies. ${ }^{34}$ In traditional methods, histological outcomes, including sparing, quantification of myelin loss and motor neuron counts, are demonstrated via staining sections of the spinal cord. ${ }^{11}$ Hematoxylin and eosin (H\&E) is a well-known general structural stain used in many tissues. Cresyl violet stains both neurons and glia and connects to acidic parts of cells such as ribosomes, nuclei and nucleoli and also demonstrates the Nissl substance. The myelin could be displayed using different techniques such as Luxol Fast Blue, Osmium Tetroxide, Eriochrome Cyanine and Kluver-Barrera or Weil. Table 3 presents the frequency and utilization of histological and chemical techniques used in the animal models of SCI classified according to MeSH terms.

Behavioral outcomes in experimental SCI models are important for evaluating the extent of injury and treatment efficacy. Activity levels are related to the extent of neuronal destruction in the gray matter at the injury region, the loss of descending and ascending axons in the white matter and the reorganization of the remaining functional nervous system. ${ }^{48}$ The locomotion could be assessed using different tests. Observing and evaluating animal activity in an open field, for example, by the Basso-Beattie-Bresnahan (BBB) score, is a simple way to assess locomotion. Table 4 presents the frequency and utilization of behavior assessment tests used in the animal models of SCI. To discern small changes in motor recovery requires more accurate and particular tests. Video recording-based kinematic analysis is one of the most sensitive behavioral evaluation tools and permits the perception of functional changes that are undetectable by visual observation alone. ${ }^{22}$ The upper limb movements could be evaluated from home-cage activity ${ }^{49}$ and reaching/grasping behavior including the food pellet reaching, the Montoya staircase and the isometric pull task..$^{50}$ The forelimb asymmetry test during vertical exploration (cylinder test) can measure the recovery of forelimb function following cervical SCI. ${ }^{11}$

Neurophysiology is a broader field that includes evoked potentials, electromyography, electroencephalography, electroneurography, membrane potentials and nerve conduction studies. These evoked potential assessments are useful for surveying the deficits and functional recovery in the neural substrates, and neural pathway integrity. ${ }^{51,52}$ Somatosensory evoked potentials (SSEP) are helpful for the assessment of sensory spinal axon conduction. ${ }^{53}$ Motor-evoked potentials offer valuable insight into the physiological status of motor tracts within the spinal cord and are appropriate for animal studies; these are a complementary for SSEP monitoring, not an alternative. All evoked potential methods take little time and cause only minimal distress or pain and can safely be done without anesthesia. ${ }^{54}$ Electromyography can be achieved both by intramedullary manipulation and rapidly applied transaxial spinal cord compression, and can be used to study autonomic dysreflexia. ${ }^{55}$ According to our data, neurophysiology was assessed in $17.8 \%$ of related studies.

Imaging. Magnetic resonance imaging (MRI) has been conveniently applied in rodent SCI models. MRI can discriminate between white and gray matter, and approximate the size of lesions and discern the formation of scars and cavities. MR images also can be used on living animals. ${ }^{22}$ Functional MRI is a precise method used to distinguish between the recovery of sensory and motor function. ${ }^{56}$

\section{Limitations}

In general, a systematic review requires a comprehensive literature involving at least two databases. However, PubMed is the most complete bibliographic database of biomedicine. ${ }^{57}$ Furthermore,
MeSH-based queries return more relevant articles compared with keyword searching and are generally recommended by librarians. ${ }^{57}$ Although it is desirable to include the greatest possible number of applicable articles, using two databases to retrieve all articles with a high yield, low specificity approach could result in an unreasonably large number of irrelevant articles at tremendous burden to the investigators. Hence a crucial balance must be found, and it does not appear that a more widespread search leads to a significant difference in the overall results. In addition, a comprehensive survey involving even unpublished work is beyond the scope of this study.

Gender and transgenic animals were not addressed in this systematic review. On the basis of the checklist of Hassannejad et al. ${ }^{9}$ about potential variables affecting the quality of animal SCI studies, gender was not included. Also, gender was not mentioned in many animal studies, especially about fish, reptiles and birds.

\section{CONCLUSION}

Animal models of SCI have been tremendously helpful for understanding of the pathophysiology, potentials for recovery, and implementations of new therapeutic strategies, and will continue to play an essential role in the investigation into such injuries. These models provide an opportunity for researchers to determine the characteristic pattern of cell death and sparing, and measurement of any demyelination, collateral sprouting, regeneration, neuroprotection and recovery of locomotor or other deficits. However, prior to choosing an animal model, the goals and objectives of the proposed study must precisely be defined. Various injury mechanisms and study designs can be useful depending on the information that is desired. Contusion and compression models better simulate the biomechanics and neuropathology of human injury. Transection models are valuable for studying anatomic regeneration. Newer SCI models require further improvement and validation. Rodents are the most commonly used and probably best-suited species for preliminary SCI studies. Controlling and monitoring injury mechanism techniques for evaluating outcomes should be continually improved as technology progresses.

\section{DATA ARCHIVING}

There were no data to deposit.

\section{CONFLICT OF INTEREST}

The authors declare no conflict of interest.

\section{ACKNOWLEDGEMENTS}

This study was a part of $\mathrm{PhD}$ thesis of the first author and was funded by Sina Trauma and Surgery Research Center, Tehran University of Medical Sciences. The authors thank Prof. Alexander R. Vaccaro (The Rothman Institute at Thomas Jefferson University, Philadelphia, PA, USA) for his precious edit of the manuscript, Dr Ayat Ahmadi (Research Development Center, Sina Hospital, Tehran University of Medical Sciences) for performing the statistical analyses, Farhad Shokraneh for his valuable comments in the search strategy, and Dr Mina Tajvidi, Masoud Hassanabadi and Dr Seyedmehdi Nourashrafeddin for providing full-texts.

1 Blight AR. Animal models of spinal cord injury. Top Spinal Cord Inj Rehabil 2000; 6 : $1-13$.

2 Blight AR, Tuszynski MH. Clinical trials in spinal cord injury. J Neurotrauma 2006; 23: 586-593.

3 Akhtar AZ, Pippin JJ, Sandusky CB. Animal models in spinal cord injury: a review. Rev Neurosci 2008; 19: 47-60. 
4 Cheriyan T, Ryan DJ, Weinreb JH, Cheriyan J, Paul JC, Lafage V et al. Spinal cord injury models: a review. Spinal Cord 2014; 52: 588-595.

5 Filli L, Schwab ME. The rocky road to translation in spinal cord repair. Ann Neurol 2012; 72: 491-501.

6 Battistuzzo CR, Callister RJ, Callister R, Galea MP. A systematic review of exercise training to promote locomotor recovery in animal models of spinal cord injury. J Neurotrauma 2012; 29: 1600-1613.

7 Nakae A, Nakai K, Yano K, Hosokawa K, Shibata M, Mashimo T. The animal model of spinal cord injury as an experimental pain model. J Biomed Biotechnol 2011; 2011: 939023.

8 Moher D, Liberati A, Tetzlaff J, Altman DG. Preferred reporting items for systematic reviews and meta-analyses: the PRISMA statement. Int J Surg 2010; 8: 336-341.

9 Hassannejad Z, Sharif-Alhoseini M, Shakouri-Motlagh A, Vahedi F, Zadegan SA Mokhatab $\mathrm{M}$ et al. Potential variables affecting the quality of animal studies regarding pathophysiology of traumatic spinal cord injuries. Spinal Cord 2015; 54: 579-583.

10 Ferguson AR, Irvine KA, Gensel JC, Nielson JL, Lin A, Ly J et al. Derivation of multivariate syndromic outcome metrics for consistent testing across multiple models of cervical spinal cord injury in rats. PLOS ONE 2013; 8: e59712.

11 Gensel JC, Tovar CA, Hamers FP, Deibert RJ, Beattie MS, Bresnahan JC. Behavioral and histological characterization of unilateral cervical spinal cord contusion injury in rats. J Neurotrauma 2006; 23: 36-54.

12 Soblosky JS, Song J-H, Dinh DH. Graded unilateral cervical spinal cord injury in the rat: evaluation of forelimb recovery and histological effects. Behav Brain Res 2001; 119 $1-13$.

13 Rahimi-Movaghar V. Clinical trials for the treatment of spinal cord injury: cervical and lumbar enlargements versus thoracic area. Brain 2009; 132: e115.

14 Lane MA, Fuller DD, White TE, Reier PJ. Respiratory neuroplasticity and cervical spinal cord injury: translational perspectives. Trends Neurosci 2008; 31: 538-547.

15 Goshgarian HG, Koistinen JM, Schmidt ER. Cell death and changes in the retrograde transport of horseradish peroxidase in rubrospinal neurons following spinal cord hemisection in the adult rat. J Comp Neurol 1983; 214: 251-257.

16 Pearse DD, Lo TP Jr, Cho KS, Lynch MP, Garg MS, Marcillo AE et al. Histopathological and behavioral characterization of a novel cervical spinal cord displacement contusion injury in the rat. J Neurotrauma 2005; 22: 680-702.

17 Ohta K, Fujimura Y, Nakamura M, Watanabe M, Yato Y. Experimental study on MRI evaluation of the course of cervical spinal cord injury. Spinal Cord 1999; 37: 580-584

18 Dunham KA, Siriphorn A, Chompoopong S, Floyd CL. Characterization of a graded cervical hemicontusion spinal cord injury model in adult male rats. J Neurotrauma 2010; 27: 2091-2106.

19 Baussart B, Stamegna JC, Polentes J, Tadie M, Gauthier P. A new model of upper cervical spinal contusion inducing a persistent unilateral diaphragmatic deficit in the adult rat. Neurobiol Dis 2006; 22: 562-574.

20 Nobunaga Al, Go BK, Karunas RB. Recent demographic and injury trends in people served by the model spinal cord injury care systems. Arch Phys Med Rehabil 1999; 80 1372-1382.

21 Vijayaprakash K, Sridharan N. An experimental spinal cord injury rat model using customized impact device: a cost-effective approach. J Pharmacol Pharmacother 2013; 4: 211-213

22 Brösamle C, Huber AB. Cracking the black box-and putting it back together again: animal models of spinal cord injury. Drug Discov Today Dis Models 2007; 3: 341-347.

23 Rahimi-Movaghar V, Yazdi A, Karimi M, Mohammadi M, Firouzi M, Zanjani LO et al. Effect of decompression on complete spinal cord injury in rats. Int J Neurosci 2008; 118: 1359-1373.

24 Jazayeri SB, Firouzi M, Abdollah Zadegan S, Saeedi N, Pirouz E, Nategh M et al. The effect of timing of decompression on neurologic recovery and histopathologic findings after spinal cord compression in a rat model. Acta Med Iran 2013; 51: 431-437.

$25 \mathrm{Lim} \mathrm{JH}$, Jung CS, Byeon YE, Kim WH, Yoon JH, Kang KS et al. Establishment of a canine spinal cord injury model induced by epidural balloon compression. J Vet Sci 2007; 8: 89-94.

26 Dimar JRI, Glassman SD, Raque GH, Zhang YP, Shields CB. The influence of spinal canal narrowing and timing of decompression on neurologic recovery after spinal cord contusion in a rat model. Spine 1999; 24: 1623.

27 Kundi S, Bicknell R, Ahmed Z. Spinal cord injury: current mammalian models. Am J Neurosci 2013; 4: 1-12.

28 Lavrov I, Gerasimenko YP, Ichiyama RM, Courtine G, Zhong H, Roy RR et al. Plasticity of spinal cord reflexes after a complete transection in adult rats: relationship to stepping ability. J Neurophysiol 2006; 96: 1699-1710.

29 Courtine G, Gerasimenko Y, van den Brand R, Yew A, Musienko P, Zhong $\mathrm{H}$ et al. Transformation of nonfunctional spinal circuits into functional states after the loss of brain input. Nat Neurosci 2009; 12: 1333-1342.
30 Shah PK, Gerasimenko Y, Shyu A, Lavrov I, Zhong H, Roy RR et al. Variability in step training enhances locomotor recovery after a spinal cord injury. Eur J Neurosci 2012; 36: 2054-2062.

31 Edgerton VR, Tillakaratne NJ, Bigbee AJ, de Leon RD, Roy RR. Plasticity of the spinal neural circuitry after injury. Annu Rev Neurosci 2004; 27: 145-167.

32 Schwab ME, Bartholdi D. Degeneration and regeneration of axons in the lesioned spinal cord. Physiol Rev 1996; 76: 319-370.

33 Steward O, Zheng B, Tessier-Lavigne M. False resurrections: distinguishing regenerated from spared axons in the injured central nervous system. J Comp Neurol 2003; 459: $1-8$.

34 Talac R, Friedman JA, Moore MJ, Lu L, Jabbari E, Windebank AJ et al. Animal models of spinal cord injury for evaluation of tissue engineering treatment strategies. Biomaterials 2004; 25: 1505-1510.

35 Lang-Lazdunski L, Matsushita K, Hirt L, Waeber C, Vonsattel J-PG, Moskowitz MA Spinal cord ischemia: development of a model in the mouse. Stroke 2000; 31: 208-213.

36 Gaviria M, Haton H, Sandillon F, Privat A. A mouse model of acute ischemic spinal cord injury. J Neurotrauma 2002; 19: 205-221.

37 Nouri M, Rasouli M, Shafiei S, Tavasoly A, Dehpour AR, Rahimi-Movaghar V. Does abdominal aorta clamping, as a method of spinal ischemia in rats, really work? Surg Neurol 2006; 66: 332-333.

38 Yezierski RP, Liu S, Ruenes GL, Kajander KJ, Brewer KL. Excitotoxic spinal cord injury: behavioral and morphological characteristics of a central pain model. Pain 1998; 75: 141-155.

39 Piao MS, Lee JK, Jang JW, Kim SH, Kim HS. A mouse model of photochemically induced spinal cord injury. J Korean Neurosurg Soc 2009; 46: 479-483.

40 Masri R, Quiton RL, Lucas JM, Murray PD, Thompson SM, Keller A. Zona incerta: a role in central pain. J Neurophysiol 2009; 102: 181-191.

41 Simmons D. The use of animal models in studying genetic disease: transgenesis and induced mutation. Nat Educ 2008; 1: 70.

42 Friedli L, Rosenzweig ES, Barraud Q, Schubert M, Dominici N, Awai L et al. Pronounced species divergence in corticospinal tract reorganization and functional recovery after lateralized spinal cord injury favors primates. Sci Transl Med 2015; 7: 302 ra134.

43 Doyle LMF, Stafford PP, Roberts BL. Recovery of locomotion correlated with axona regeneration after a complete spinal transection in the eel. Neuroscience 2001; 107: 169-179.

44 Lee D-H, Lee J. Animal models of axon regeneration after spinal cord injury. Neurosci Bull 2013; 29: 436-444.

45 Sharif-Alhoseini M, Rahimi-Movaghar V. in Topics in Paraplegia. (ed. Dionyssiotis Y) 209-228 (InTechOpen, 2014).

46 Jakeman L, Ma M, Stokes B. Considering the Use of Transgenic Mice in Spinal Cord Injury Research. In: Marwah J, Dixo E, Banik N (eds). Traumatic CNS Injury. Prominent Press: New York, USA, 2001, pp 180-202.

47 Pilcher HR. It's a knockout. Nature 2003; 425: 570-571.

48 Basso DM. Behavioral testing after spinal cord injury: congruities, complexities, and controversies. J Neurotrauma 2004; 21: 395-404.

49 Wu R, Su L, Yang PF, Min Chen L. Altered spatiotemporal dynamics of cortical activation to tactile stimuli in somatosensory area $3 \mathrm{~b}$ and area 1 of monkeys after spinal cord injury. eNeuro. (e-pub ahead of print 29 Sep 2016. doi:10.1523/ENEURO.0095-16.2016).

50 Fenrich KK, May Z, Torres-Espín A, Forero J, Bennett DJ, Fouad K et al. Single pellet grasping following cervical spinal cord injury in adult rat using an automated full-time training robot. Behav Brain Res 2016; 299: 59-71.

51 Šedý J, Urdzíková L, Jendelová P, Syková E. Methods for behavioral testing of spinal cord injured rats. Neurosci Biobehav Rev 2008; 32: 550-580.

52 Onifer SM, Rabchevsky AG, Scheff SW. Rat models of traumatic spinal cord injury to assess motor recovery. ILAR J 2007; 48: 385-395.

53 Onifer SM, Zhang YP, Burke DA, Brooks DL, Decker JA, McClure NJ et al. Adult rat forelimb dysfunction after dorsal cervical spinal cord injury. Exp Neurol 2005; 192 : 25-38.

54 Blight AR. Motor evoked potentials in CNS trauma. Cent Nerv Syst Trauma 1986; 3 : 207-214.

55 Skinner SA, Transfeldt EE. Electromyography in the detection of mechanically induced spinal motor tract injury: observations in diverse porcine models. J Neurosurg Spine 2009; 11: 369-374.

56 Li S, Kim JE, Budel S, Hampton TG, Strittmatter SM. Transgenic inhibition of Nogo-66 receptor function allows axonal sprouting and improved locomotion after spinal injury. Mol Cell Neurosci 2005; 29: 26-39.

57 Kim S, Yeganova L, Wilbur WJ. Meshable: searching PubMed abstracts by utilizing MeSH and MeSH-derived topical terms. Bioinformatics 2016; 32: 3044-3046.

Supplementary Information accompanies this paper on the Spinal Cord website (http://www.nature.com/sc). 